To appear: Lund, K., Rosseti, C., Metz, S. (forthcoming). Do internal factors of cooperation influence computer-mediated distance activity? In the proceedings of the international conference CSCL 2007, July 16-July 21, New Brunswick, New Jersey, USA.

\title{
Do internal factors of cooperation influence computer-mediated distance activity?
}

\author{
Kristine Lund, Céline Rossetti, Stéphanie Metz, \\ ICAR, CNRS, University of Lyon, 15 parvis René Descartes, BP 700069342 Lyon Cedex, \\ Email \{Kristine.Lund, Celine.Rossetti, Stephanie.Metz\}@univ-lyon2.fr
}

\begin{abstract}
On the one hand, researchers have studied factors that influence collaboration and on the other, researchers have proposed models of collaborative problem solving. However, we have not found research on the relation between these factors and the dimensions used in order to describe the collaborative activity within the models. This article's goal is to propose such relations for a situation of collaborative design, mediated by computer and carried out at a distance. We will show two main relations that emerged from our corpus. Firstly, dialogue utterances between partners that have a dominant social aspect are positively related to the symmetry of the entire dyadic interaction in terms of partners' contributions. Secondly, dialogue utterances that predominantly deal with expressing what partners are doing is negatively related to the extent to which partners are aligned. This research also extends the field of applicability of the cooperative activity model proposed in Baker (2002).
\end{abstract}

\section{Introduction}

The models of cooperation elaborated in domains dealing with problem resolution aided by computers focus on a variety of phenomena. For example, they address spatial aspects of virtual worlds (Benford, Bullock, Cook, Harvey, Ingram \& Lee (1993), decision processes carried out through a distributed information system (Gachet \& Haettenschwiler, 2003) or forms of cooperation as reflected by dyadic interactions (Baker, 2002).

This last model of cooperation — the only full-fledged model amongst those cited above — is interesting in that it distinguishes different forms of cooperation within a dyad. Making these forms explicit helps us to understand how participants organize themselves when they solve a problem together. This organization is a function of the dimensions of activity that make up the model. From the moment that these forms are defined, it becomes possible to evaluate which of them characterize those interactions that are favorable for learning (Jakobsson, 2002; Burton, Brna \& Treasure-Jones, 1996; Baker, 2002). Alternatively, it becomes possible to study which forms of cooperation would produce a final product of greater quality, for example in the case of design. But on which basis should we choose the dimensions of activity that are used to elaborate the forms of cooperation? And what factors can be related to these dimensions and in consequence, influence the forms of cooperation?

\section{A model of cooperative activity and factors influencing cooperation}

In the conceptual model of cooperative activity during problem resolution described by Baker, (2002), three principal phenomena appear upon observing people that work together: 1) different responsibilities and contributions, 2) the way in which people carry out their work together (e.g. each person's speed, mutual understanding), and finally 3 ) the presence or absence of agreement. The objective of Baker's model is to establish a link between cooperative activity and learning through the combination of three fundamental dimensions: role symmetry, alignment and agreement. (cf. Table 1).

Table 1. The fundamental dimensions of Baker's (2002) model of forms of cooperation

\begin{tabular}{|l|l|}
\hline Dimension & Definition \\
\hline Degree of symmetry & $\begin{array}{l}\text { For a given continuous sequence of interaction, the similarity or difference in the responsibilities of } \\
\text { participants in regards to the accomplishment of sub-tasks, such as is manifested in verbal or non-verbal } \\
\text { communication, linked to material resources. }\end{array}$ \\
\hline Degree of & The difference in propositional attitudes (belief, non-belief, acceptance, non-acceptance) that are \\
\hline
\end{tabular}




\begin{tabular}{|l|l|}
\hline agreement & $\begin{array}{l}\text { manifested publicly, in relation to the different aspects of cooperative activity of problem solving } \\
\text { (solutions, goals, methods, actions). }\end{array}$ \\
\hline Degree of alignment & $\begin{array}{l}\text { The extent to which partners are "in phase", in relation to aspects of their cooperative problem solving } \\
\text { (phases, degree of mutual comprehension, conceptualization of problem). }\end{array}$ \\
\hline
\end{tabular}

In the case where the values of the dimensions are binary (e.g. symmetrical / non-symmetrical, etc.), the three dimensional space corresponds to eight specific forms of cooperation (cf. Figure 1).

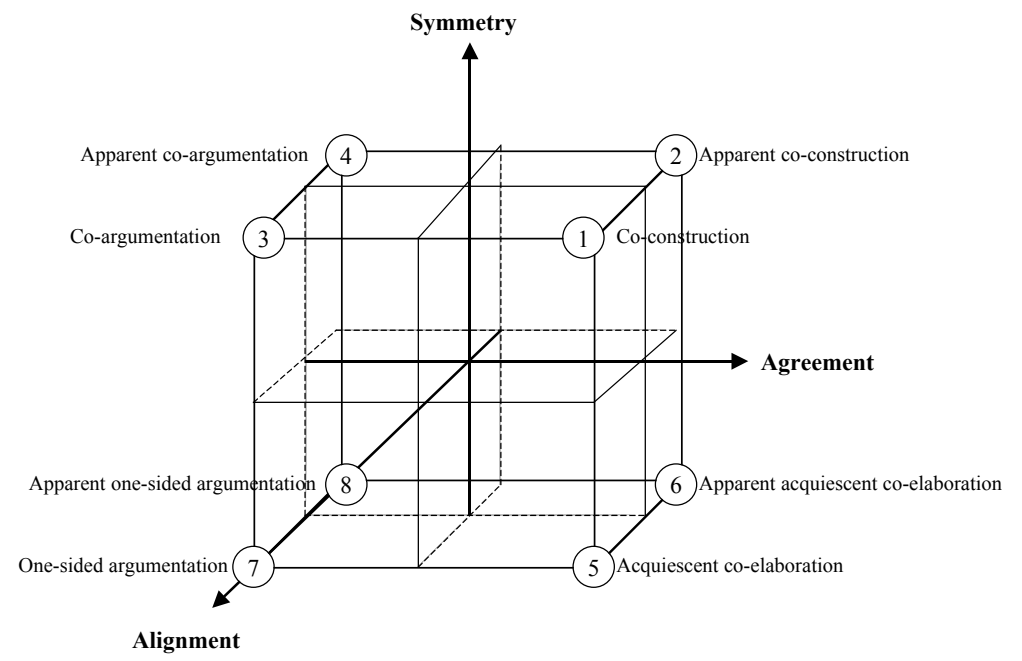

Figure 1. The eight basic forms of cooperation in the cooperative activity of problem resolution, redrawn from Baker (2002).

A study of the literature revealed factors that contribute in diverse ways to collaboration. However, they are not put into relation with a model that describes the forms of collaborative activity through precise dimensions, such as those in Baker's model (see above). This is the objective of our article. We organized the factors we found in the literature into two types: internal and external. Each type of factor contains examples of different granularity. In regards to internal factors, a factor can be internal to the individual or internal to the interaction between individuals.

The factors that are internal to the individual are for example, self-efficacy (Bandura, 1994), adherence to the working principle, altruism or favorable opinion of collaboration as an approach to problem solving (Suangsuwan, Wiratchai, \& Wongwanich, 2006). There are also numerous factors internal to the interaction between individuals. For example, the structuring of communication at a distance (Baker \& Lund, 1997), the socioinstitutional roles of participants (Lund, 2003), the exchanges of a social nature at the beginning of an interaction (van Amelsvoort \& Andriessen 2003), and finally the extent to which participants take into account non-verbal body language (Gregori \& Brassac, 2001) are all correlated with diverse ways of describing how collaboration occurs.

The external factors of a technological, cultural, organizational, physiological and economical type can also influence the ways actions are carried out in collaborative and distributed communities (Strauss (1993), cited by Fjuk \& Dirckinck-Homfeld, 1997). Concerning finer grained external factors, the availability of material resources can influence the way in which collaboration evolves: (Scott, Mandryk \& Inkpen, 2002). In addition, the control of a resource (e.g. the manipulation of a video) can be determined by the seat one chooses (Krafft \& Dausendschön-Gay, 1999).

The first type of factor (internal) and more specifically, the factors that are internal to the interaction between individuals will retain our attention for the research presented here. In what follows, we apply Baker's model (2002) to two new corpora, gathered in two design situations (a pilot study and a principal experiment), both mediated by computer and at a distance. The dimensions that constitute the forms of cooperative activity (symmetry, accord \& alignment) are distinguished according to a methodology adapted to our corpora and from this, we deduce a subset of possible forms of cooperative activity. An analysis of the first corpus identified three factors internal to the interaction between individuals that were correlated with different forms of cooperation in Baker's model. These 
correlations were tested on the second corpus. In the following sections, we present our methodology, our results and interpretations. Finally, we present our conclusions and our perspectives for this research.

\section{Methodology, analyses and results}

In this section we briefly describe our pilot study and show how the results enabled us to form hypotheses about the correlation of specific forms of collaborative activity with three factors internal to interaction between individuals. These hypotheses are tested in the principal study, also described below.

\section{Pilot study}

Here, we describe the participants, their prescribed task, the resources they had at their disposal, the experimental protocol, the corpus obtained, the analyses carried out (coding of interactions and determination of forms of cooperative activity), our results and finally our observations and hypotheses.

\section{Participants, task and resources}

Six university students, non-experts of origami, but experienced in working on computers, formed the three observed dyads. The participants were asked to compose a procedural text for folding an origami paper hen. They carried out this task on the Internet, each on his or her own computer. The participants did not know each other before the experiment and were asked not to attempt to identify their partner, once they connected. Lack of available computer rooms meant that in reality, participants were all in the same computer room, although they were told they were each connected to a person in another location.

The partners had instructions on paper that they could consult at any time. They also had three tools on their computer screen, two of which are included within DREW (1) (Corbel, Girardot, Jaillot, 2002; Corbel, et. al., 2003). Figure 2 shows:

1. At the top left: a video of the origami folding task that the participants can play at their leisure. The participants could fast forward, rewind or define a particular time stamp in order to get to any part in the video.

2. At the right: a shared text editor (part of DREW) in which the participants composed their procedural text for folding the origami hen. Both partners could write in the text editor, but not simultaneously, due to single cursor. When one person writes, the other sees the text appear in real time.

3. At the bottom left: a chat area with a personal text entry zone (also part of DREW). Pressing the Enter key sends the typewritten text to the shared chat board, thus rendering it visible to ones partner.

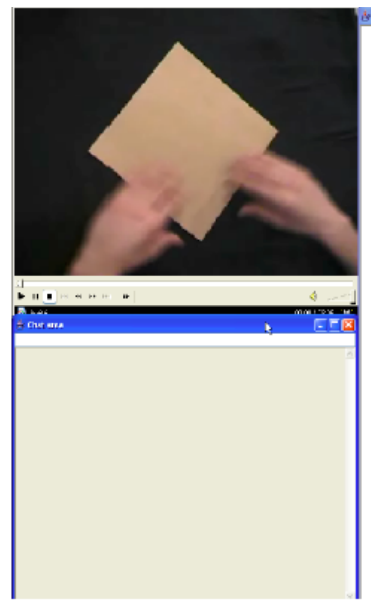

\section{Experimental protocol}

Figure 2. The video and two modules of the platform DREW.

The pilot study took place in three stages. First, an experimenter explained the task to the participants and described the tools they could use $(5 \mathrm{~min})$. Then, the participants were taken into the computer room. While they 
carried out the task (1h), 3 experimenters observed their actions in order to characterize their activity and to pinpoint any problems. At the end of the experiment, an experimenter interviewed each dyad in order to gather their impressions and to identify any difficulties linked to the resources or the experimental protocol (10-15 min).

\section{Corpus}

The participants' computer-mediated interaction via the DREW platform (chat and text editor) was traced and converted from XML into Excel ${ }^{\mathrm{TM}}$ format. The chat messages were saved each time the Enter key was pressed after a message was typed. In regards to the text editor, a first level of granularity saved the totality of the text, approximately every second. This was difficult to analyze (cf. Figure 3). A second level of granularity was thus created (Dyke, 2006). Rather than send the entire contents of the text editor to the server, only the modified line (which was given a number) and its state before the modification were saved (cf. Figure 4). A line of text is sent to the server in three cases: 1) when there is no activity in the text editor for more than 5 seconds (= production: timeout), 2) when the participant changes to the chat (= production: speaker changed module) or 3) when ones partner starts composing in the text editor at the same time (= production: speaker was interrupted).

\begin{tabular}{llll}
\hline Time & Participant & Action in the interface & Module \\
\hline $14: 27: 7$ & Aldébaran & ok let's start & chat \\
$14: 27: 13$ & Bételgeuse & ok, so this should work & chat \\
$14: 27: 18$ & Bételgeuse & trial2 & textboard \\
$14: 27: 22$ & Bételgeuse & In ord & textboard \\
$14: 27: 23$ & Bételgeuse & in order & textboard \\
$14: 27: 24$ & Bételgeuse & in order to & textboard \\
$14: 27: 25$ & Bételgeuse & in order to do & textboard \\
\hline
\end{tabular}

Figure 3. An extract of the interaction (translated from the French) before modification of the trace

\begin{tabular}{|c|c|c|c|}
\hline Time & Participant & Action in the interface & Module \\
\hline $14: 27: 13$ & Bételgeuse & ok, so this should work & chat \\
\hline \multirow[t]{2}{*}{$14: 27: 18$} & Bételgeuse & $* *$ begins writing $* *$ & textboard \\
\hline & & $\begin{array}{l}1<\text { trial } 21>\text { in order to do an origami hen you need a piece of paper } 2<\text { and now } \\
\text { do you see something }\end{array}$ & \\
\hline $14: 27: 43$ & Bételgeuse & $* * *$ production: speaker was interrupted & textboard \\
\hline $14: 27: 44$ & Aldébaran & $* *$ begins writing $* *$ & textboard \\
\hline $14: 27: 44$ & Aldébaran & $* * *$ production: speaker was interrupted & textboard \\
\hline $14: 27: 44$ & Bételgeuse & $* *$ begins writing $* *$ & textboard \\
\hline $14: 27: 54$ & Bételgeuse & $\begin{array}{l}1<\text { in order to do an origami hen you need a piece of paper } 1>\text { in order to do an } \\
\text { origami hen you need a piece of paper in a square format*** production: timeout }\end{array}$ & textboard \\
\hline
\end{tabular}

Figure 4. An extract of the interaction (translated from the French) after modification of the trace

\section{Analyses: two-dimensional coding method}

A two-dimensional coding method was elaborated using the chat interventions of the pilot study as an initial corpus. We first determined the pragmatic orientation of the utterance as primarily focusing on one of four categories: the product (the written instructions for the origami hen), social aspects of discourse, the carrying out of the task and the activity (cf. Table 2 for explanations of categories and examples). We then coded each utterance according to the speech act (cf. Austin, 1962) that was used: proposition, acceptance, refusal, correction, directive, affirmative, question \& response (cf. Table 3). This allowed us to determine the cooperation as a function of the utterance's value.

Table 2. Definitions of the possible functions of an utterance and examples translated from the original French.

\begin{tabular}{|l|l|l|}
\hline & Definition & Example \\
\hline Social & $\begin{array}{l}\text { Utterances that have the function of conveying emotions and thoughts of } \\
\text { participants, expressions of politeness or any sort of utterance that does not } \\
\text { directly concern the task. }\end{array}$ & $\begin{array}{l}\text { "shall we say until our next } \\
\text { game?!" }\end{array}$ \\
\hline $\begin{array}{l}\text { Carrying out } \\
\text { the task }\end{array}$ & $\begin{array}{l}\text { The utterances that concern carrying out the task refer to the manner of } \\
\text { proceeding, for example the division of roles or procedures or strategies to } \\
\text { adopt. }\end{array}$ & $\begin{array}{l}\text { "how do you want to do this, } \\
\text { we type here, then in the text } \\
\text { area?" }\end{array}$ \\
\hline Activity & Utterances concerning "activity" are used in order to discuss what one or one's & "i'm looking at the video" \\
\hline
\end{tabular}




\begin{tabular}{|l|l|l|}
\hline & partner is doing or has done. & \\
\hline Product & $\begin{array}{l}\text { Utterances that essentially concern the content of the product (instructions for } \\
\text { folding the origami hen) as well as the description of the video. }\end{array}$ & $\begin{array}{l}\text { "then unfold again your sheet } \\
\text { of paper you should have your } \\
\text { initial sheet" }\end{array}$ \\
\hline
\end{tabular}

Table 3. Definitions of the speech act categories.

\begin{tabular}{|l|l|l|}
\hline & Definition & Example \\
\hline Proposition & $\begin{array}{l}\text { Propose, launch a debate on a proposition, either } \\
\text { implicitly or explicitly. }\end{array}$ & $\begin{array}{l}\text { "the next part : take out the end of the blue corner of } \\
\text { the center of the square that is folded in two and mark } \\
\text { the fold." }\end{array}$ \\
\hline Acceptance & Show agreement with the utterance of one's partner. & "yeah, that's perfect" \\
\hline Refusal & Show disagreement with the utterance of one's partner. & "too complicated, don't get it!" \\
\hline Correction & $\begin{array}{l}\text { Make something precise or modify a previous } \\
\text { utterance. }\end{array}$ & $\begin{array}{l}\text { "but I would add the meaning, like: the points that you } \\
\text { just folded outwards...something like that" }\end{array}$ \\
\hline Question & $\begin{array}{l}\text { An utterance that shows a request, a search for } \\
\text { understanding or for information, directed at one's } \\
\text { partner. }\end{array}$ & "how will you start?" \\
\hline Response & $\begin{array}{l}\text { An utterance that gives information in answer to a } \\
\text { previous request. }\end{array}$ & $\begin{array}{l}\text { "i'm looking at the video" (response to the above } \\
\text { example question) }\end{array}$ \\
\hline Affirmation & Give a piece of information. & "well, most of the work is coming up" \\
\hline Directive & $\begin{array}{l}\text { Not give the choice to one's partner to either refuse or } \\
\text { accept the produced utterance. Give an order or } \\
\text { obligate one's partner to carry out an action. }\end{array}$ & $\begin{array}{l}\text { "erase it if you don't like it and write something in its } \\
\text { place" }\end{array}$ \\
\hline
\end{tabular}

\section{Determining the form of cooperation}

In order to determine the form of cooperation for the different dyads, we used Baker's model of (2002), validated for pedagogical situations of cooperative problem resolution. As our situation was of another nature - a design task - we had to find a way to apply this model in our own context. We needed to find a way to characterize the three dimensions used to define forms of cooperation. Symmetry was defined according to two indicators relating to text writing, the principal task: 1) the comparison of the number of interventions in the "textboard" between the participants tells us whether the text writing is equitable; 2 ) the chat interventions of each participant concerning the text writing also allows us distinguish the transactional role of "proposer" (i.e. does one partner suggest more text proposals?). (Non)-alignment was illustrated by the (presence) absence of phrases of the type "i am lost". Granted, the absence of such phrases does not guarantee alignment: partners could agree to work on different parts of the problem. Also, partners could be aligned, but not have attained a common understanding of their work. The observation grid was also an indicator of alignment: certain partners watched the same part of the video clip at the same time and could thus orient their talk about it. Finally, agreement was decided by coding speech acts: the presence of corrections and refusals determined whether or not the dyad was generally in agreement.

\section{$\underline{\text { Results }}$}

Two of the three dyads were in complete opposition. One of them was in "co-construction": symmetrical (with equal roles), aligned (shared understanding) and in agreement (cf. Figure 5). The other dyad was in "apparent one-sided argumentation": asymmetrical (different roles for each), non-aligned (e.g. lack of shared comprehension) and in disagreement (presence of corrections or refusal of other's utterances) (cf. Figure 6). 


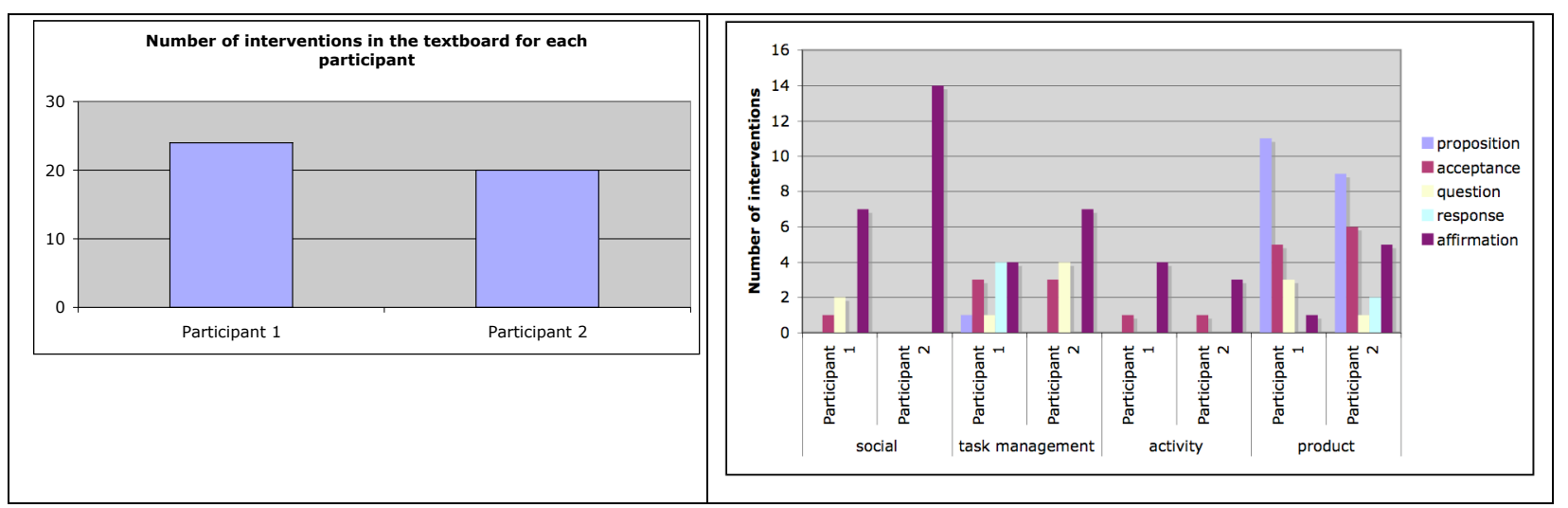

Figure 5. A dyad in co-construction

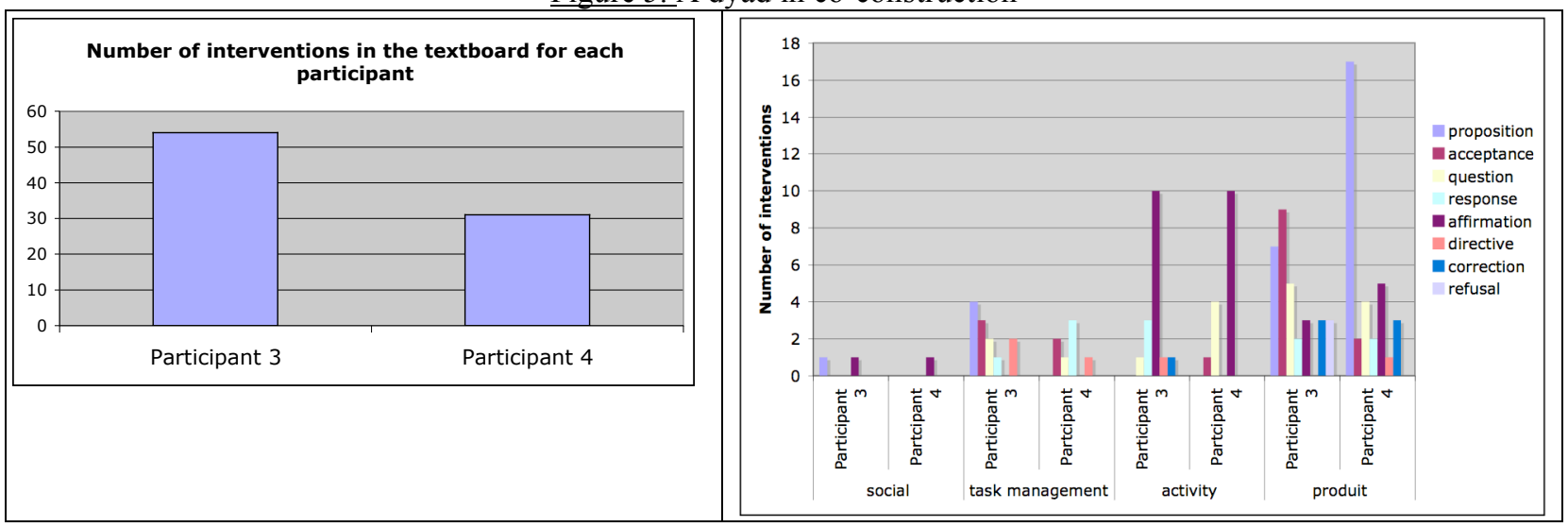

Figure 6. A dyad in apparent one-sided argumentation

\section{Observations and hypotheses}

Different observations were made by relating the forms of cooperation used by the dyads and the function of their utterances (cf. Figure 7). We can clearly see that the dyad in co-construction (symmetrical, aligned and in agreement) shows a higher proportion of socially oriented utterances. The correlation of the quantity of socially oriented utterances with good symmetry is thus the first hypothesis we sought to test. In a similar vein, we observe a higher proposition of utterances concerning carrying out the task for the dyad in construction. Our second hypothesis proposes that this type of utterances is also correlated with good symmetry.

The dyad in apparent one-sided argumentation shows a larger quantity of utterances in regards to activity than the other two dyads. This could indicate that the two actors are trying to align themselves by telling each other what they are doing in order that they may synchronize. Our third hypothesis is thus that the quantity of utterances concerning activity will be correlated to a poor alignment. 


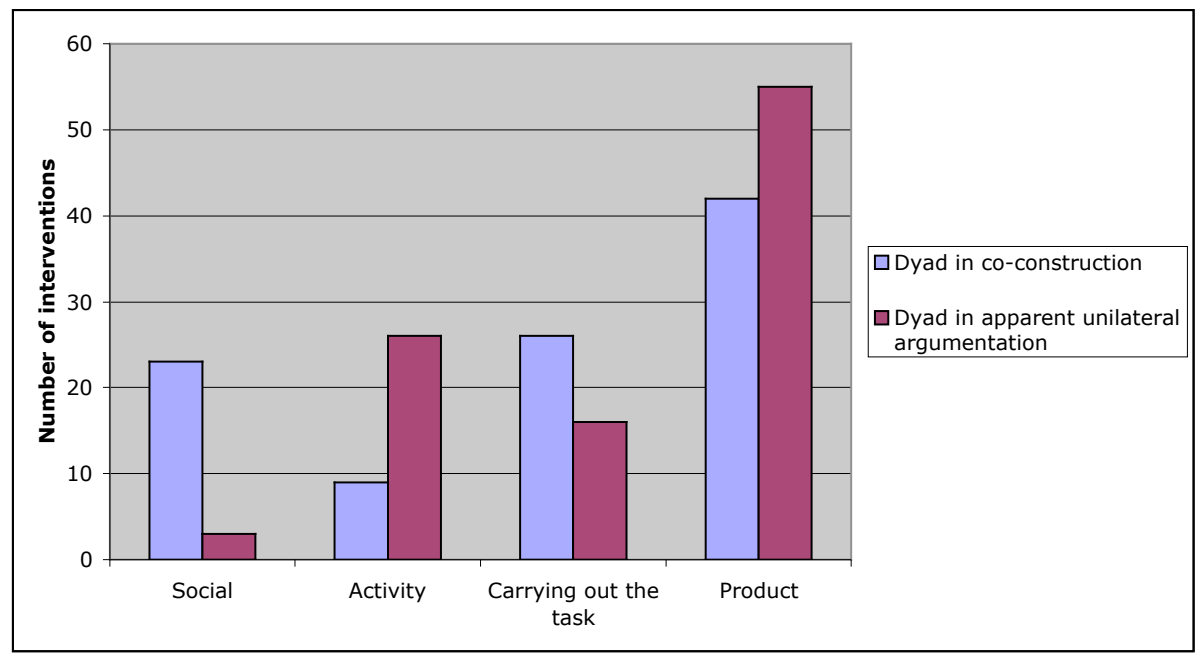

Figure 7. Percentage of utterances by function of utterance and by dyad

\section{Principal experiment}

In order to illustrate the differences with the pilot study, we present in the following sections, the participants, the material they used, the task, the experimental protocol, the corpus, the analyses carried out, the results concerning the verification of our hypotheses and finally, our interpretations.

\section{Participants, material, task and experimental protocol}

Ten dyads (twenty students aged 20-30 years) participated in the experiment. They were non-experts in origami, regularly used chat and knew how to type on a keyboard.

The material underwent a number of changes from the pilot study. The paper hen was replaced by a paper box, easier to fold in general and with less variation in difficulty. The text editor was also modified in order to allow the two participants to write simultaneously; two cursors instead of one were available. During the pilot study, the presence of one cursor created conflicts that reduced the usage of the text editor to one person at a time. Having two cursors available could favor a more symmetrical cooperation as both partners can write simultaneously (cf. Dyke, 2006). Otherwise, the platform DREW remained identical with the chat and the text editor as well as the video.

Some other modifications were made as a result of problems encountered during the pilot study. After the initial introduction $(5 \mathrm{~min})$, we added a presentation of the tools and their functions $(5 \mathrm{~min})$. In addition, a conception micro-task was inserted immediately before the task in order to familiarize the participants with the tools they were to use (10min). The questions were given in questionnaire format in order to avoid influence on the part of the experimenter, but also to obtain information that a participant may not want to divulge in front of his or her partner. (5min). Finally, an individual interview was also conducted (2min).

\section{Corpus, analyses and results}

The ten interactions of the ten dyads were automatically recorded by DREW (chat + text editor) and were transformed from XML into $\mathrm{Excel}^{\mathrm{TM}}$ format (as for the pilot study).

The same coding scheme used in the pilot study was applied to the corpus (on the chat interventions). Then, inter-coder reliability was performed on six of the ten dyadic interactions. Three different coders coded respectively three, two and one of the six interactions, depending on the time they had available. A comparison of these results was done (cf. Table 4) in relation to the original coder, who coded all ten interactions. The average of agreement of the three coders in relation to the original coder was $77.24 \%$ for functions and $71,18 \%$ for speech acts. As the percentage to be obtained in order for a coding scheme to be reliable is $70 \%$, the coding of both the utterance function and the speech acts is validated (De Wever, Schellens, Valcke \& Van Keer, 2006).

Table 4. Inter-coder reliability; Percentage of agreement with the $1^{\text {st }}$ coder, creator of the coding scheme 


\begin{tabular}{|l|l|l|l|}
\hline & Dyad name & Functions & Speech acts \\
\hline \multirow{5}{*}{$1^{\text {st }}$ coder } & Asterope-Gianfar & 75,00 & 71,71 \\
\hline \multirow{3}{*}{$2^{\text {nd }}$ coder } & Rastaban-Yildun & 80,88 & 75,73 \\
\cline { 2 - 4 } & Pleion-Wezen & 84,30 & 72,25 \\
\hline $3^{\text {rd }}$ coder & Deneb-Jabbah & 87,06 & 67,41 \\
\hline & Fornacis-Lesath & 70,83 & 73,61 \\
\hline Average of agreement & Sargas-Zibal & 65,35 & 66,34 \\
\hline
\end{tabular}

As for the pilot study, and using the same indicators, we were able to identify many forms of cooperation: three dyads in "apparent co-argumentation" (symmetrical, non-aligned, in disagreement), four dyads in "apparent one-sided argumentation" (asymmetrical, non-aligned, in disagreement), two dyads in "co-argumentation (symmetrical, aligned, in disagreement) and one dyad in "one-sided argumentation" (asymmetrical, aligned, in disagreement). The graphs below show (a)symmetry and (dis)agreement and illustrate two examples of the differences between a symmetrical and a asymmetrical dyad. (cf. Figure 8 and Figure 9).

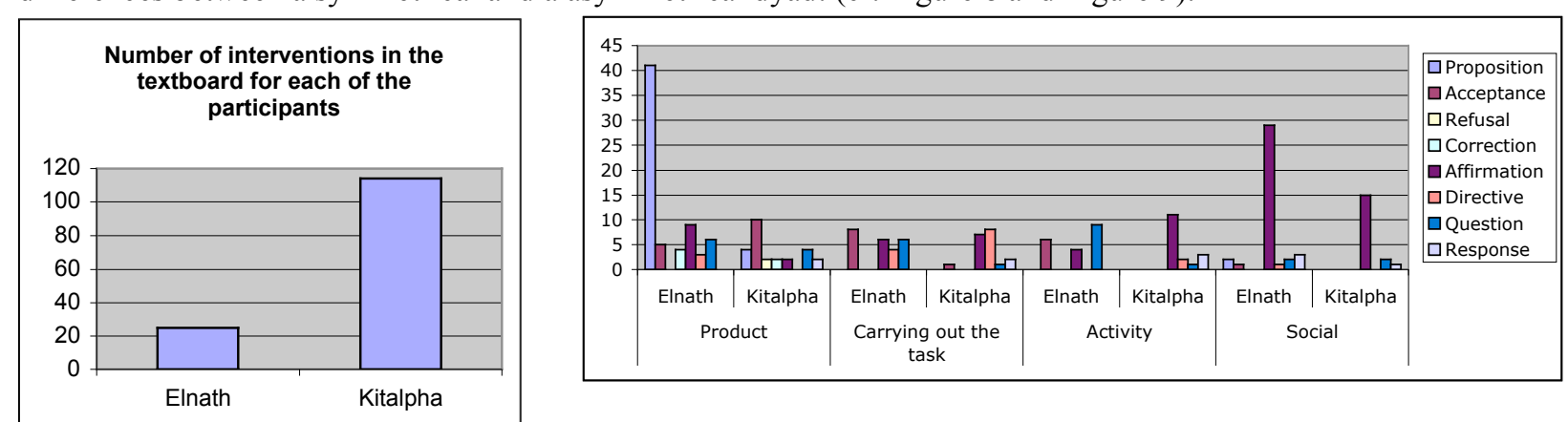

Figure 8. Example of a dyad in apparent one-sided argumentation (asymmetrical)
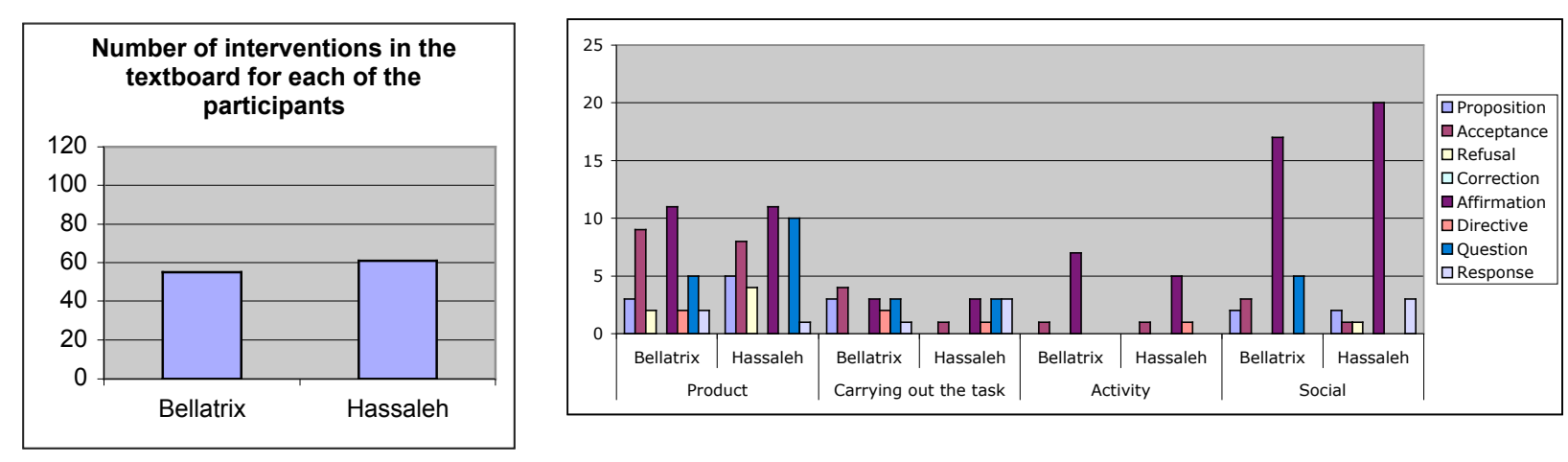

Figure 9. Example of a dyad in apparent co-argumentation (symmetrical)

Once the forms of cooperation were determined, we calculated 1) the average percentage of the utterances concerning activity according to whether the dyad was aligned or not and 2) the average percentage of the utterances concerning carrying out the task on the one hand and socially oriented utterances on the other according to whether the dyad was symmetrical or not. (cf. Figure 10). In order to ascertain whether the differences between groups were significant, we performed the non-parametric Wilcoxon test, considered to be the equivalent of the t-test.

Firstly, the quantity of socially oriented utterances is twice as large within the interactions of symmetrical dyads than for asymmetrical dyads. The Wilcoxon test shows that this difference is significant and that the two 
groups are different $(\mathrm{Ws}=16, \mathrm{p}=0.01)$. Secondly, the quantity of utterances concerning activity is almost twice as large within the interactions of non-aligned dyads than for aligned dyads. The Wilcoxon test shows that this difference is significant and that the two groups are different (Ws=7, $\mathrm{p}=0.025)$. Finally, the quantity of utterances concerning carrying out the task did not differ according to dyad symmetry. According to the Wilcoxon test, the two groups are identical $\left(\mathrm{Ws}_{\mathrm{s}}=28, \mathrm{p}>0.1\right)$.
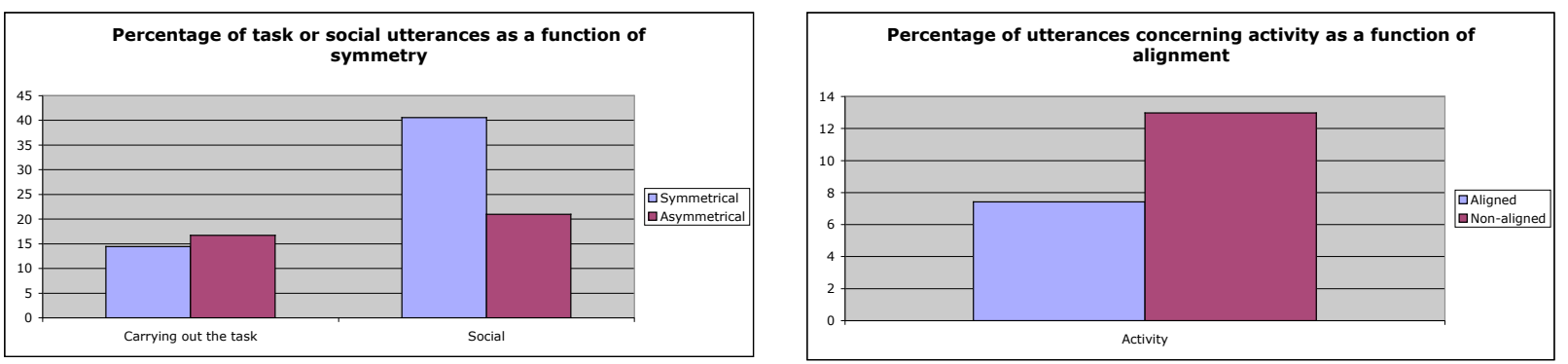

Figure 10. Cross between utterance function and the value of Baker's (2002) dimensions of cooperative problem solving: alignment and symmetry

\section{$\underline{\text { Interpretations }}$}

Contrary to the data from the pilot study, no dyad was in agreement. We notice much more argumentation on the content of the instructions the students wrote. Perhaps the presence of two cursors in the text editor allowed participants to write what they wanted without first expressing themselves in the chat module and this created disagreement. The presence of one cursor obligates the two participants to reflect together on the content of the instructions before writing them. Viewing a larger number of dyads allowed us to see that being aligned at a distance is rather rare (seven dyads out of ten are not aligned) Not perceiving certain actions of ones partner and not being able to establish eye-contact with him or her does not help in synchronizing reflections and actions.

In regards to the link between (a)symmetry and the quantity of socially oriented utterances (1st hypothesis), it is possible that maintaining good social rapport with ones partner allows for a relation of equality that translates into equitable transactional roles, assuming similar socio-institutional roles. The interactions of symmetrical dyads contain a higher quantity of socially oriented utterances than the interactions of asymmetrical dyads.

However, whatever the form of cooperation, it seems necessary that dyads exchange utterances dealing with carrying out the task. Our 2nd hypothesis is thus not confirmed. There is no link between symmetry and utterances about task. At first glance, this is contrary to the research of Lahti, Seitamaa-Hakkarainen \& Hakkarainen, (2001). They have found differences in the proportion of the interaction consecrated to the organization of a design task according to group. Indeed, it seems that groups that "collaborate" discuss in greater length the organization of their design than the groups that "cooperate" or simply "coordinate". Their work environment has more modules that allow for more of a difference in task organization according to group. Certain modules are more pertinent than others in carrying out the task and this leads to choosing amongst them. On the other hand, our own participants only had to organize the use of the textboard and this module is inherent to the writing of the instructions and thus must necessarily be managed.

The link between (non)-alignment and the quantity of utterances concerning activity shows that the lack of information emanating from ones partner concerning his or her activity is compensated for by transmitting this information in writing (chat messages). This was our 3rd hypothesis. When partners are not aligned, it seems they seek to synchronize themselves by expressing what they are doing or by asking what their partner is doing. This is shown by the higher quantity of utterances concerning the activity than partners who are aligned.

\section{Conclusions and perspectives}

Our work has enlarged the field of applicability of Baker's model (2002); we have shown it is possible to apply this model with success to computer-mediated interactions whose objective is the design of a procedural text (instructions for origami folding). We have also established a correlation between certain dimensions of this model and three factors internal to dyadic interaction. Firstly, there is a greater quantity of socially oriented utterances in symmetrical interactions than in asymmetrical interactions. Secondly, there is a greater quantity of utterances 
dealing with activity (e.g. i'm looking at the video) in non-aligned interactions than in aligned interactions. Thirdly, whatever the cooperation type, and considering that all dyads were in disagreement, there is a high quantity of utterances that deal with carrying out the task.

In these two studies (pilot and principal), only the chat messages were analyzed for content. In the near future, we would like to analyze the contributions made to the text editor from two angles: the type of contribution (new production, correction, etc.) and the participant who contributed. This would allow us to refine the notion of symmetry in relation to the whole task. It would also be interesting to relate these observations to the quality of the product in order to appreciate which form of cooperation is optimal.

\section{Endnotes}

(1) DREW (Dialogical Reasoning Educational Webtool) was designed and developed during the European project SCALE, (Internet-based intelligent tool to Support Collaborative Argumentation-based LEarning in secondary schools, 2001-2004), 5th Framework, IST (Internet Societies Technologies).

\section{References}

Austin, J. L. (1962). How to do things with words. Cambridge, MA: Harvard University Press.

Baker, M.J. (2002). Forms of cooperation in dyadic problem-solving. Revue d'Intelligence Artificielle, 16 (4-5), 587620 .

Baker, M.J. \& Lund, K. (1997). Promoting reflective interactions in a computer-supported collaborative learning environment. Journal of Computer Assisted Learning. 13, 175-193.

Bandura, A. (1994). Self-efficacy. In V. S. Ramachaudran (Ed.), Encyclopedia of human behavior, Vol. 4, (pp. 7181). New York: Academic Press.

Benford, S. D., Bullock, A. N., Cook, N. L., Harvey, P. W., Ingram, R. J. and Lee, O., (1993). A Spatial Model of Cooperation for Virtual Worlds. In Proceedings of Informatique '93: Interfaces to Real and Virtual Worlds, Montpelier, France, March 1993.

Burton, M., Brna, P. and Treasure-Jones, T. (1997). Splitting the Collaborative Atom: How to Support Learning about Collaboration. In du Boulay, B. and Mizoguchi, R. (Eds.) Artificial Intelligence in Education: Knowledge and Media in Learning Systems, 135-142. IOS, Amsterdam.

Corbel, A., Girardot, J.J., Jaillon, P. (2002). DREW: A Dialogical Reasoning Web Tool, In Proceedings of ICTE2002, International Conference on ICT's in Education. Badajoz, Espagne, November 13-16, 2002.

Corbel, A., Jaillon, P., Serpaggi, X., Baker, M., Quignard, M., Lund, K., (2003). DREW : Un outil internet pour créer des situations d'apprentissage coopérant [DREW : An internet tool for creating co-operative learning situations]. In Desmoulins, Marquet, \& Bouhineau (Eds.), Proeedings of EIAH2003 Environnements Informatiques pour l'Apprentissage Humain (pp. 109-113). Strasbourg.

De Wever, B., Schellens, T., Valcke, M., Van Keer, H. (2006). Content analysis schemes to analyze transcripts of online asynchronous discussion groups: A review. Computers \& Education, 46(1) 6-28.

Dyke, G. (2006). Extension of the Musette framework for synchronous collective activities. Unpublished Masters Thesis, EPFL, Lausanne, Switzerland.

Fjuk, A., Dirckinck-Holmfeld, L. (1997). Articulation of Actions in Distributed Collaborative Learning. Scandinavian Journal of Information Systems, 9(2), 3-24.

Gachet, A.et Haettenschwiler, P. (2003). Distributed Decision Support Systems - A Federalist Model of Cooperation, in Bisdorff R. (ed.) Human Centered Processes - Distributed Decision Making and ManMachine Cooperation, proceedings of the 14th MINI EURO Conference Luxembourg,( pp. 211-216).

Grégori, N. et Brassac, C. (2001). La conception collaborative d'artefacts : activités cognitives en situation dialogique [The collaborative design of artifacts: cognitive activities in dialogical situations] In ÉPIQUE, Journées d'étude en psychologie ergonomique, Nantes, October 29-30, 2001, pp. 21-31.

Jakobsson, A. (2002). Learning Attitudes Decisive to Students Cognitive and Knowledge Development, In ICCE02, International Conference on Computers in Education, p. 1025.

Krafft, Ulrich \& Dausendschön-Gay, (1999). Système écrivant et processus de mise en mots dans les rédactions conversationnelles [Writing system and the process of putting into words in conversational writing]. Langages, 134, 51-67.

Lahti, H., Seitamaa-Hakkarainen, P., \& Hakkarainen, K. (2001). The nature of collaboration in computer-supported designing. In P. Dillenbourg, A. Eurelings., \& K. Hakkarainen (Eds.), Proceedings of European 
Perspectives on Computer-Supported Collaborative Learning. (pp. 381-388). Maastricht, the Netherlands: Maastricht McLuhan Institute.

Lund, K. Analyse de l'activité explicative en interaction : étude de dialogues d'enseignants de physique en formation interprétant les interactions entre élèves [The analysis of interactive explanatory activity: a study of physics student teachers' dialogues as they interpret student interactions. Ph.D., Université J. Fourier, 2003.

Scott, S. D., Mandryk, R. L, \& Inkpen, K. M. (2002). Understanding children's interactions in synchronous shared environments, Proceedings of Computer Supported Collaborative Learning (CSCL). Boulder, CO, USA. January 2002. Mahwah, NJ: Lawrence Erlbaum Associates Inc.

Strauss, A. (1993). Continual Permutations of Actions. Aldine de Gruyter, New York.

Suangsuwan, J., Wiratchai, N., \& Wongwanich, S. A Development of indicators, and the Cause and Effect Model of Collaboration of Primary School Teacher in Ayutthaya province, Thailand. Downloaded April 28, 2006 from http://www.aare.edu.au/05pap/sua05024.pdf

Van Amelsvoort, M. \& Andriessen, J. Is the tone set? How the start of the social relation between two students defines the content collaboration. Downloaded April 28, 2006 from http://edu.fss.uu.nl/ord/fullpapers/Amelsvoort-Erkens.doc,

\section{Acknowledgements}

We would like to thank Jean-Laurent Cassier, John Bouvery and Magalie Ollagnier-Beldame for their work in carrying out inter-coder reliability. 